\title{
WIN2021
}

University of Minnesota, USA

June, 2021

Virtual Conference

QED radiative corrections

to charged-current neutrino-nucleon elastic scattering

for accelerator neutrino experiments

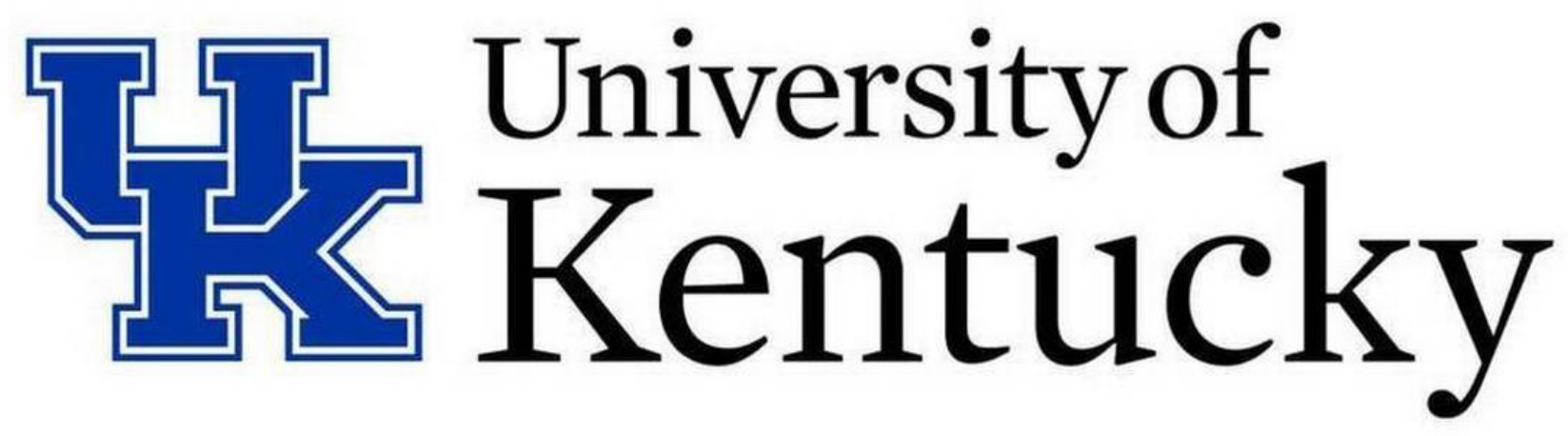

Oleksandr Tomalak

O.T., Qing Chen, Richard J. Hill and Kevin S. McFarland, arXiv: 2105.07939 


\section{Neutrino experiments}

- DUNE and Hyper-K: leading-edge $v$ science experiments

\section{Sanford Underground}

Research Facility
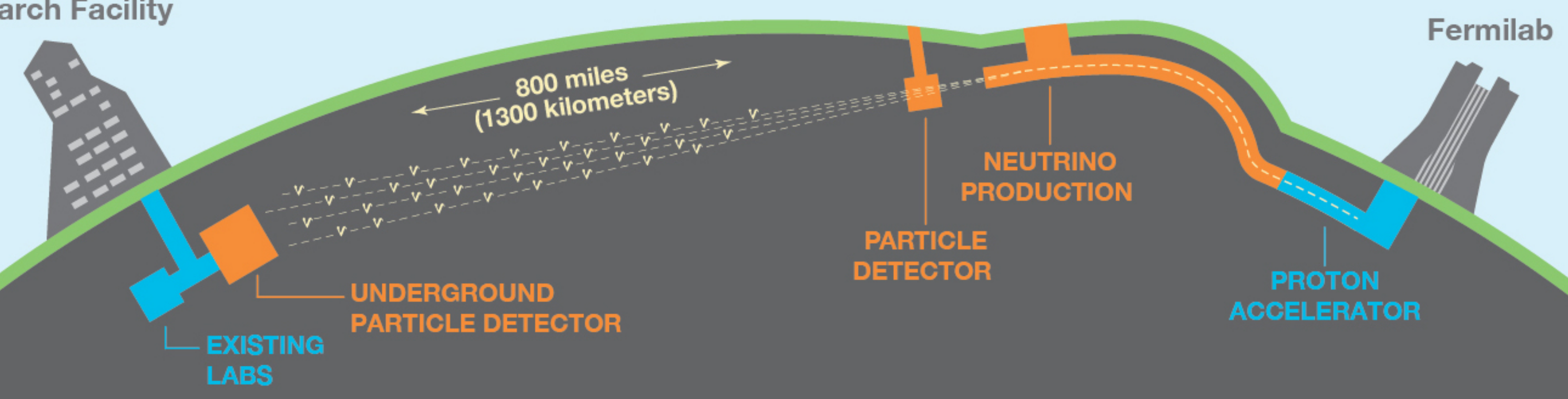

- measurement of $v_{\mu}$ disappearance and $v_{\mathrm{e}}$ appearance

$$
N_{\nu} \sim \int \mathrm{d} E_{\nu} \Phi_{\nu}\left(E_{\nu}\right) \times \sigma\left(E_{\nu}\right) \times R\left(E_{\nu}, E_{\nu}^{\mathrm{rec}}\right)
$$

- near detector: determine flux and cross sections 
Neutrino interactions

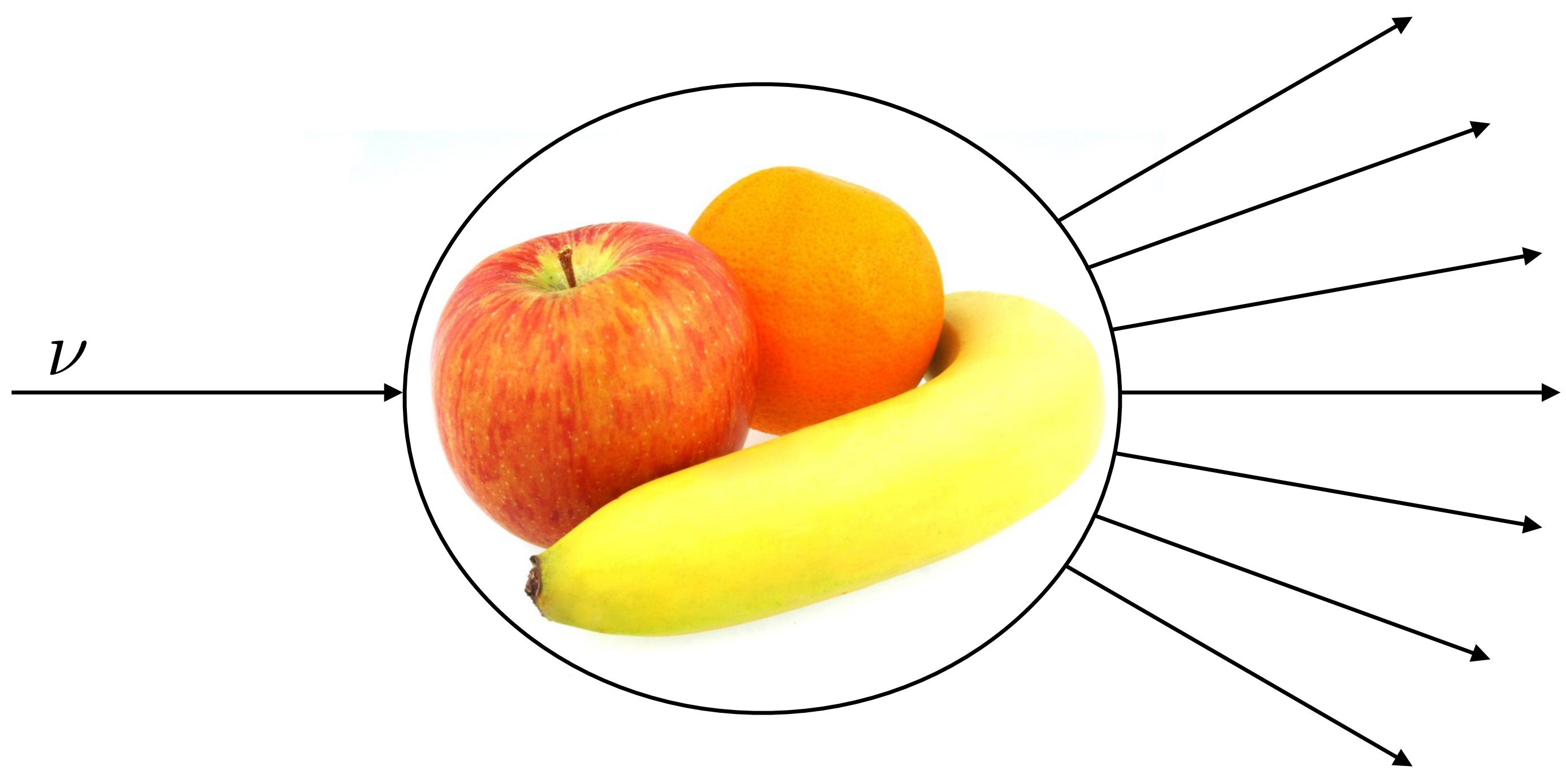




\section{QED corrections}

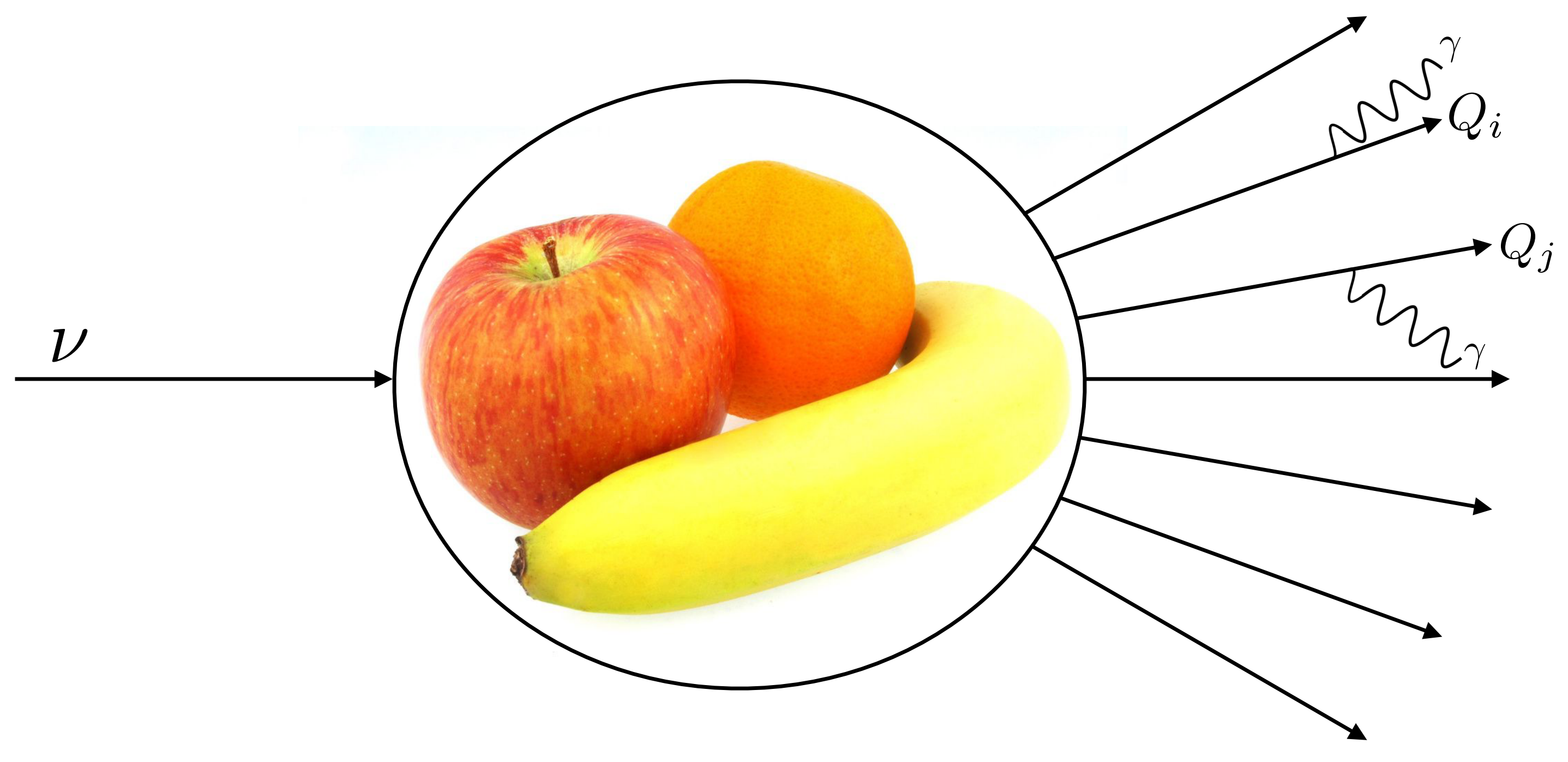

- all charged particles couple to real and virtual photons 


\section{QED corrections}

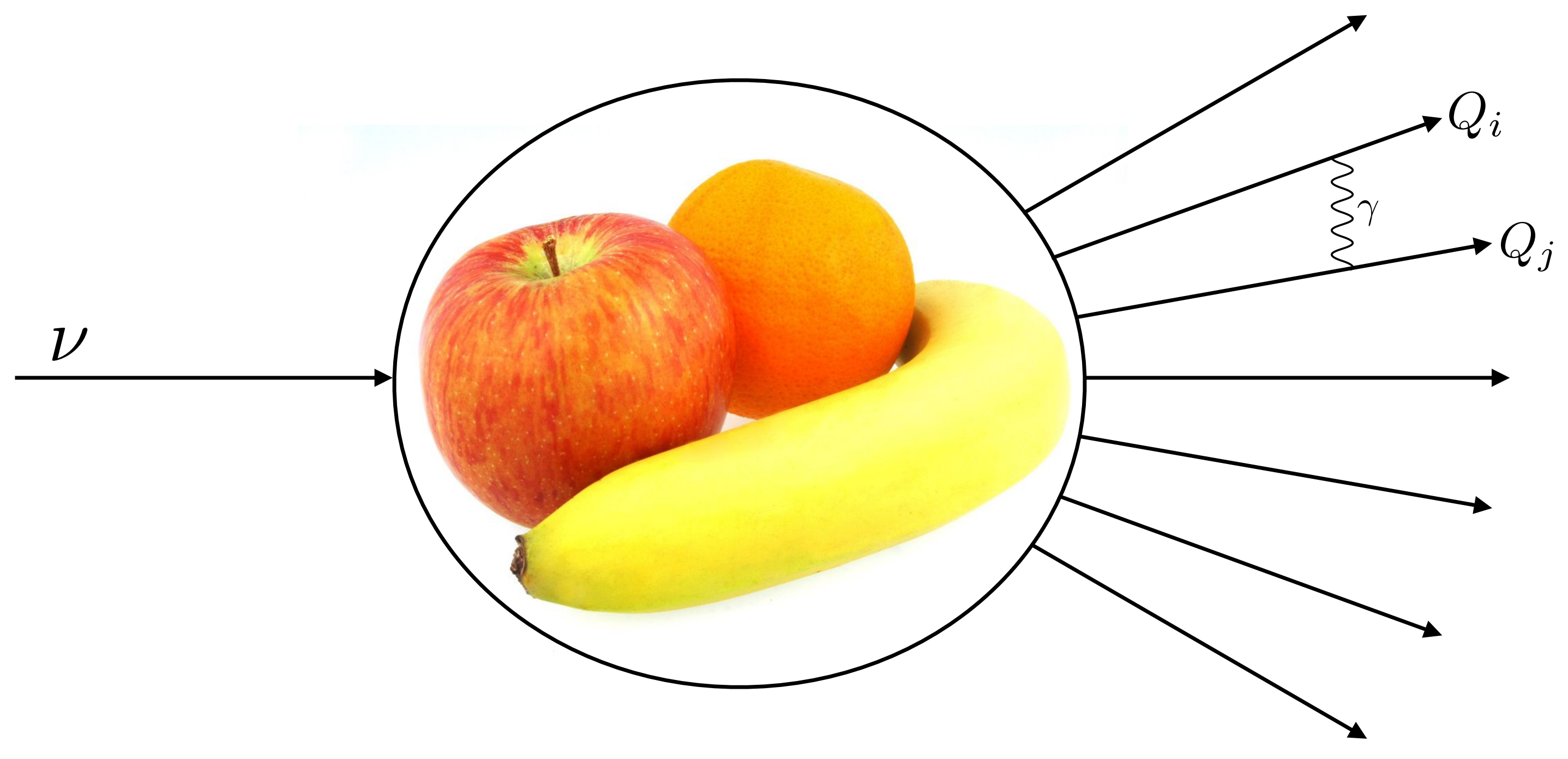

- all charged particles couple to real and virtual photons 


\section{QED corrections}

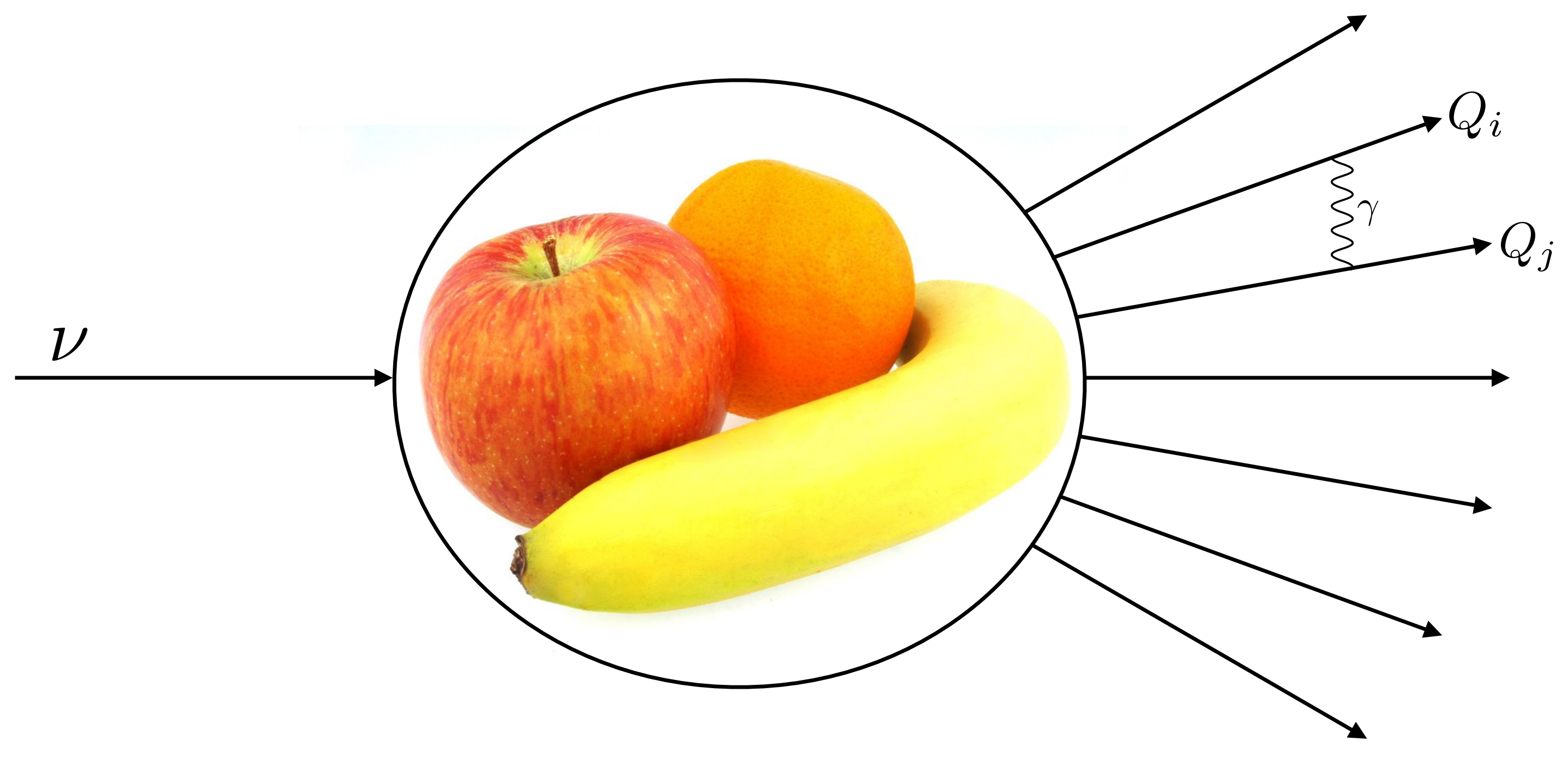

$-\frac{\alpha}{\pi} \sim 0.2 \%$ suppression by electromagnetic coupling constant 


\section{QED corrections}

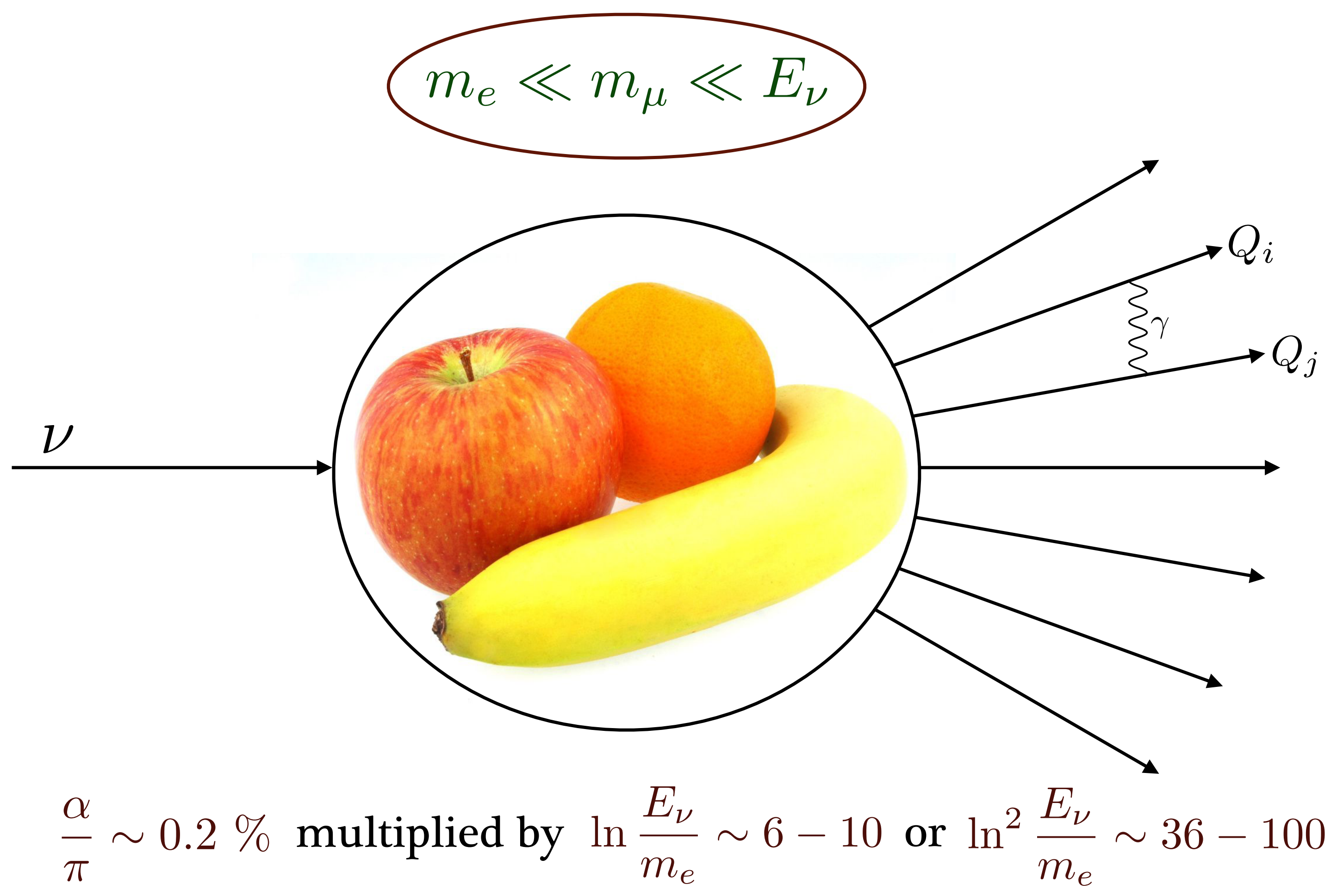

- scale separation: large flavor-dependent logarithms for GeV energy 


\section{CCQE. Why should we care?}

- neutrino-nucleus cross sections and future accelerator-based fluxes

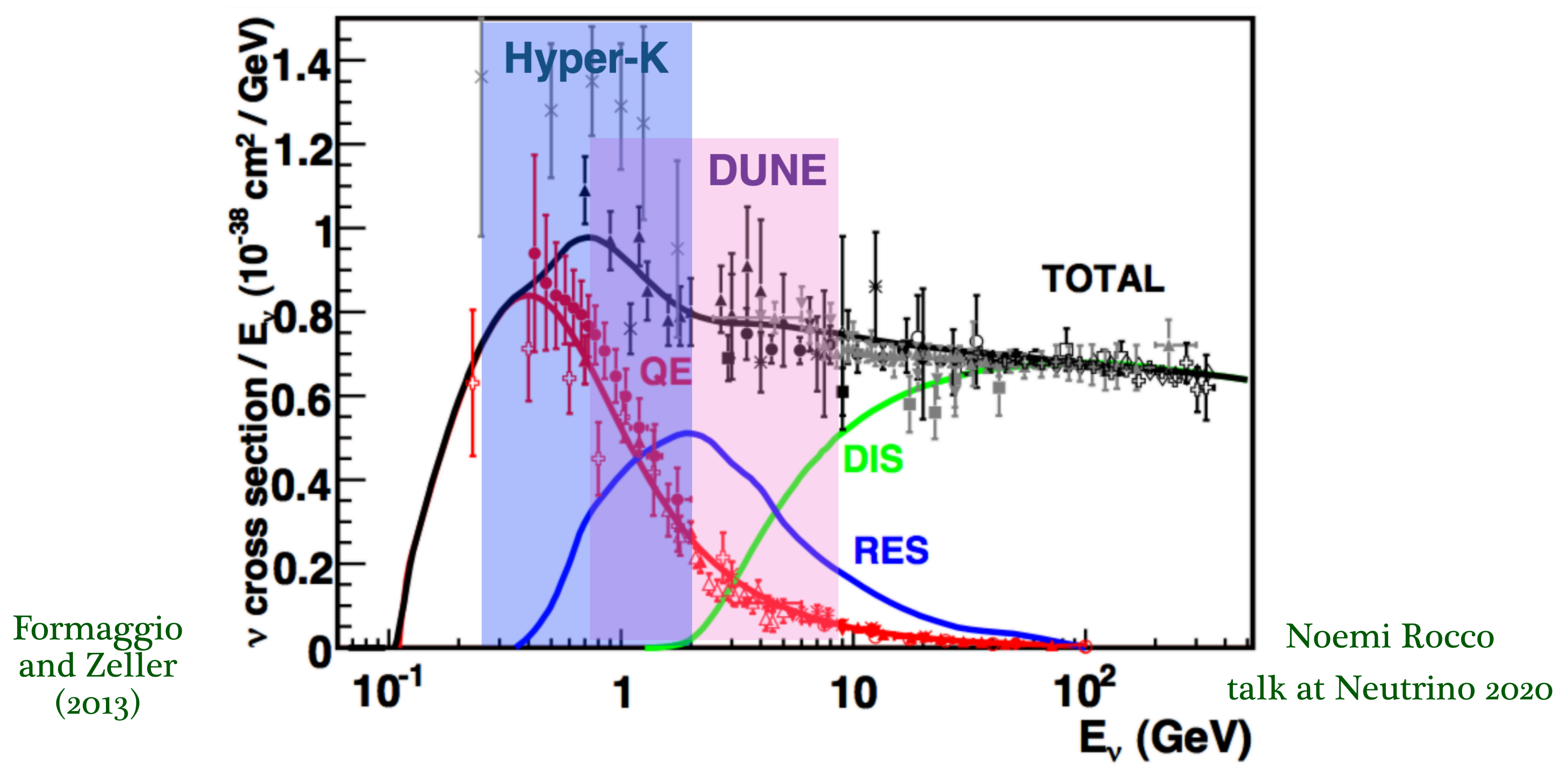

- basic process: bulk of events at Hyper-K and DUNE

- best channel for reconstruction of neutrino energy 


\section{Scattering on free nucleon}

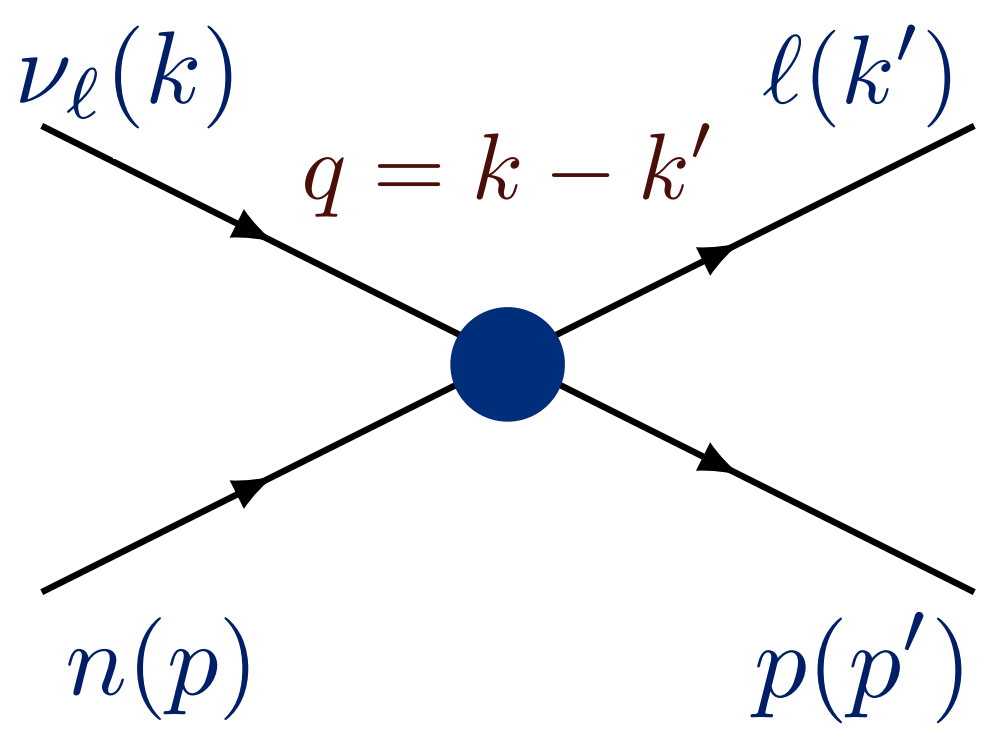

neutrino energy

momentum transfer

$Q^{2}=-q^{2}$

contact interaction at $\mathrm{GeV}$ energies

- assuming isospin symmetry, nucleon current:

$$
\Gamma^{\mu}\left(Q^{2}\right)=<p\left|\bar{u}\left(\gamma^{\mu}-\gamma^{\mu} \gamma_{5}\right) d\right| n>
$$

$$
\Gamma^{\mu}\left(Q^{2}\right)=\gamma^{\mu} F_{D}^{V}\left(Q^{2}\right)+\frac{i \sigma^{\mu \nu} q_{\nu}}{2 M} F_{P}^{V}\left(Q^{2}\right)+\gamma^{\mu} \gamma_{5} F_{A}\left(Q^{2}\right)+\frac{q^{\mu}}{M} \gamma_{5} F_{P}\left(Q^{2}\right)
$$

form factors: isovector Dirac and Pauli axial and pseudoscalar

$$
F_{D, P}^{V}=F_{D, P}^{p}-F_{D, P}^{n}
$$

tree-level amplitude

$$
\mathrm{T}=\frac{\mathrm{G}_{\mathrm{F}} V_{u d}}{\sqrt{2}}\left(\bar{\ell}\left(k^{\prime}\right) \gamma_{\mu}\left(1-\gamma_{5}\right) \nu_{\ell}(k)\right)\left(\bar{p}\left(p^{\prime}\right) \Gamma^{\mu}\left(Q^{2}\right) n(p)\right)
$$




\section{Radiative corrections}

- large kinematic logarithms enhance radiative corrections

$$
\frac{\alpha}{\pi} \sim 0.2 \% \quad \text { multiplied by } \quad \ln \frac{E_{\nu}}{m_{e}} \sim 6-10
$$

- cross sections with electron flavor are subject to large corrections

- phase-space restrictions enhance radiative corrections

$$
\begin{array}{lll}
\frac{\alpha}{\pi} \sim 0.2 \% & \text { multiplied by } & \ln ^{2} \frac{E_{\nu}}{m_{e}} \sim 36-100 \\
E_{\gamma}<\Delta E & \text { soft photons } & 2 \ln \frac{E_{\nu}}{m_{e}} \ln \frac{\Delta E}{m_{e}} \sim 35-60
\end{array}
$$

smaller collinear logarithms

- crucial dependence on detector details

- radiative corrections crucial for \%-level oscillation program 


\section{Static nucleon limit}

- formal limit of infinitely heavy nucleus

- provides correct soft and collinear logarithms

- soft-photon energy $<20 \mathrm{MeV}$, jet size: $10^{\circ}$ for electron and $2^{\circ}$ for muon
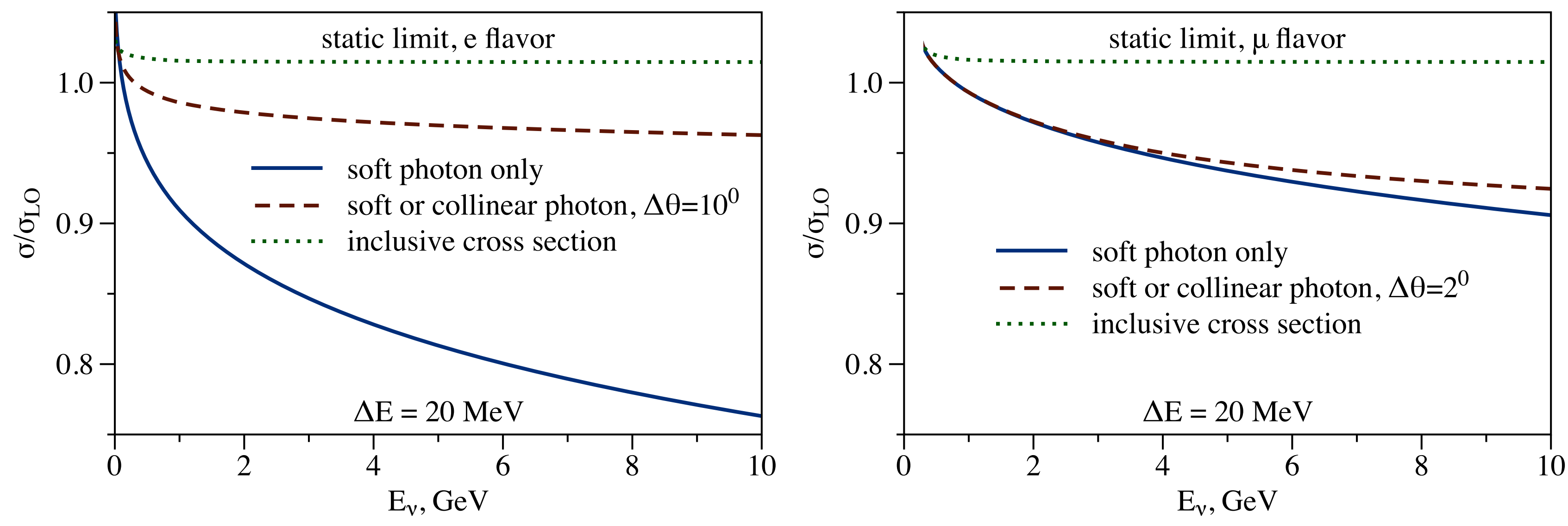

- flavor-dependent effect, same for $\nu_{\ell} n \rightarrow \ell^{-} p$ vs $\bar{\nu}_{\ell} p \rightarrow \ell^{+} n$

- collinear observable: cancellation of virtual vs real logs

- inclusive observables $(+\gamma)$ : few \% level, flavor independent 


\section{Electron vs muon jets}

- factorization for radiation of collinear photons

- cone angle is defined to lepton direction

- photons of energy $>20 \mathrm{MeV}$, fixed energy in the cone
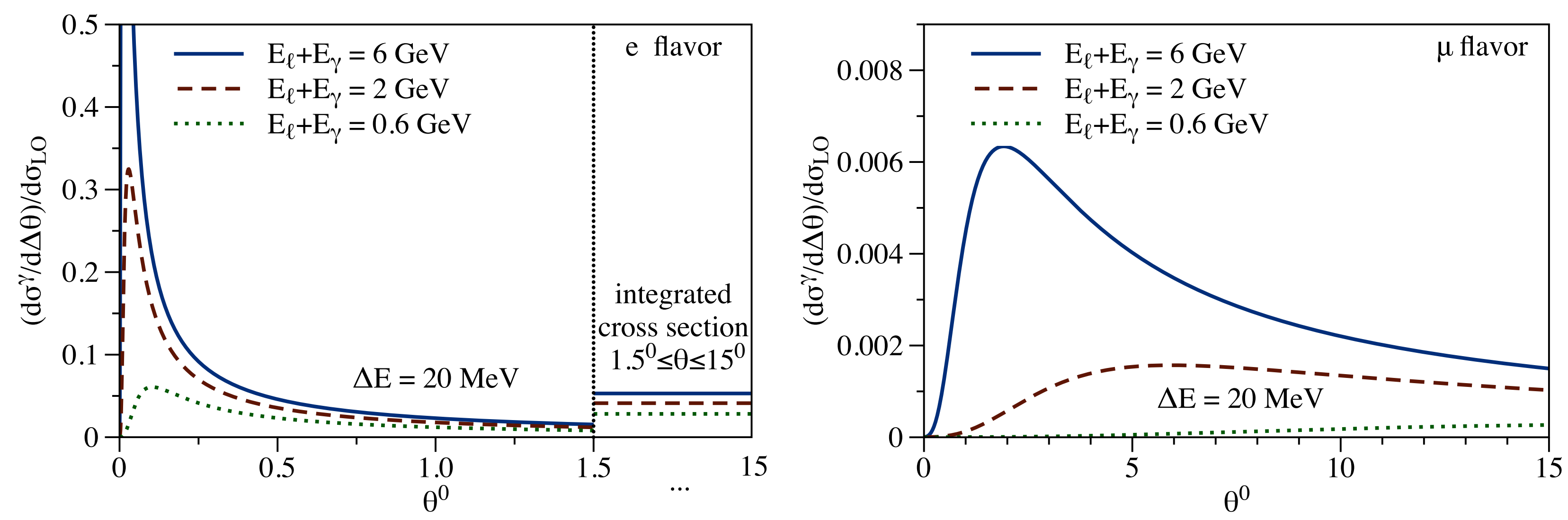

- flavor-dependent effect, same for $\nu_{\ell} n \rightarrow \ell^{-} p$ vs $\bar{\nu}_{\ell} p \rightarrow \ell^{+} n$

- forward-peaked radiation for electron flavor

- negligible radiation for muons with shifted peak position 


\section{Factorization approach}

- cross section is given by factorization formula

$$
\mathrm{d} \sigma \sim S\left(\frac{\Delta E}{\mu}\right) J\left(\frac{m_{\ell}}{\mu}\right) H\left(\frac{M}{\mu}\right)
$$

-M - determine hard function at hard scale by matching experiment or hadronic model to the theory with heavy nucleon

- soft and collinear functions are evaluated perturbatively 


\section{Hadronic model at $\mathrm{GeV}$ scale}
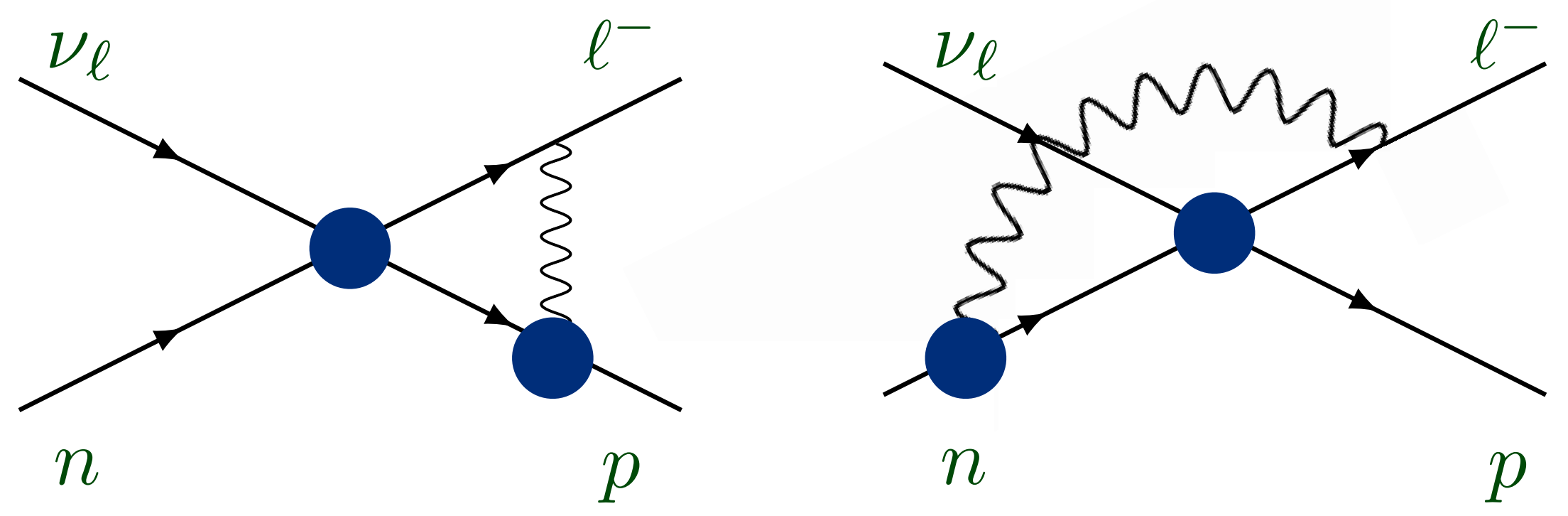

- exchange of photon between the charged lepton and nucleons

- assume onshell form for each interaction with dipole form factors discussed for neutrino-nucleon scattering: Graczyk (2013)

- add self energy for charged particles

- reproduce soft and collinear regions of SCET

- best determination of hard function 


\section{Factorization approach}

- cross section is given by factorization formula

$$
\mathrm{d} \sigma \sim S\left(\frac{\Delta E}{\mu}\right) J\left(\frac{m_{\ell}}{\mu}\right) H\left(\frac{M}{\mu}\right)
$$

- determine hard function at hard scale by matching experiment or hadronic model to the theory with heavy nucleon

- RGE evolution of the hard function to scales $\Delta E, m_{\ell}$

- soft and collinear functions are evaluated perturbatively

- $m_{e}$ - calculate cross section at low energies accounting for all large logs ep scattering with soft radiation only: Richard J. Hill (2016)

- soft and collinear functions determined analytically

- hard function describes physics at GeV energies 


\section{Error budget}

- uncertainties from hard function
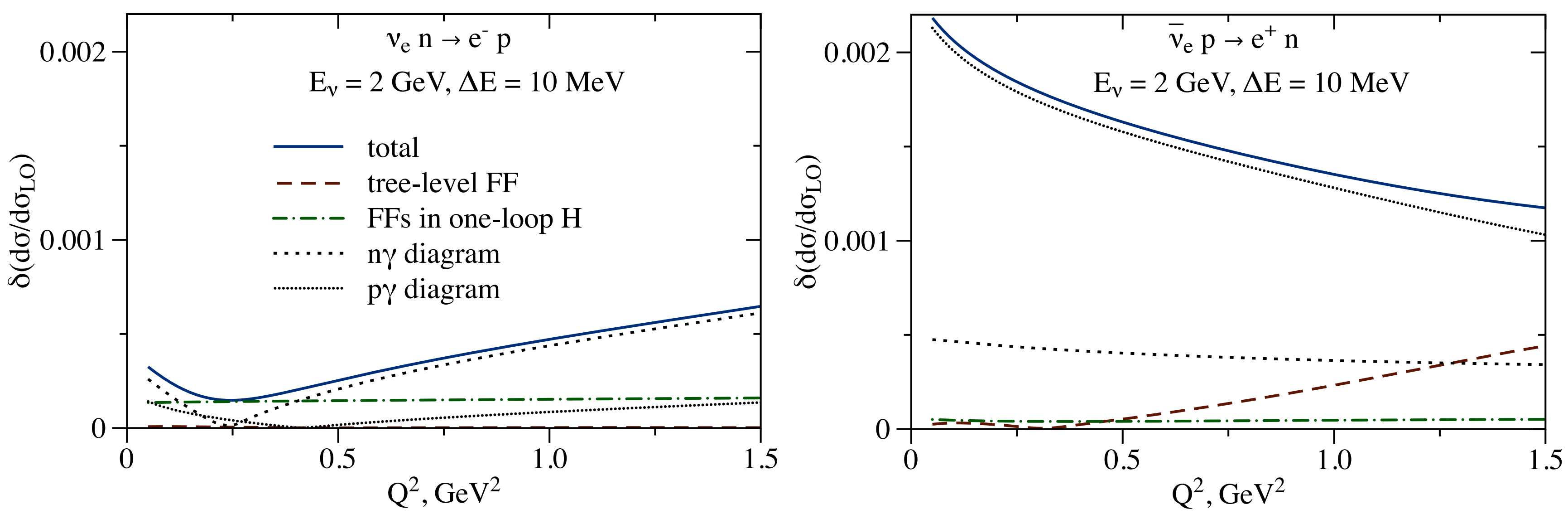

- nucleon form factors

Meyer, Betancourt, Gran and Hill (2016) Kaushik Borah, Gabriel Lee, Richard J. Hill and O.T. (2020)

- add perturbative uncertainty by variation of scale

- uncertainty of permille level for the ratio to LO result 


\section{Cross section. Electron flavor}

- exclusive observables
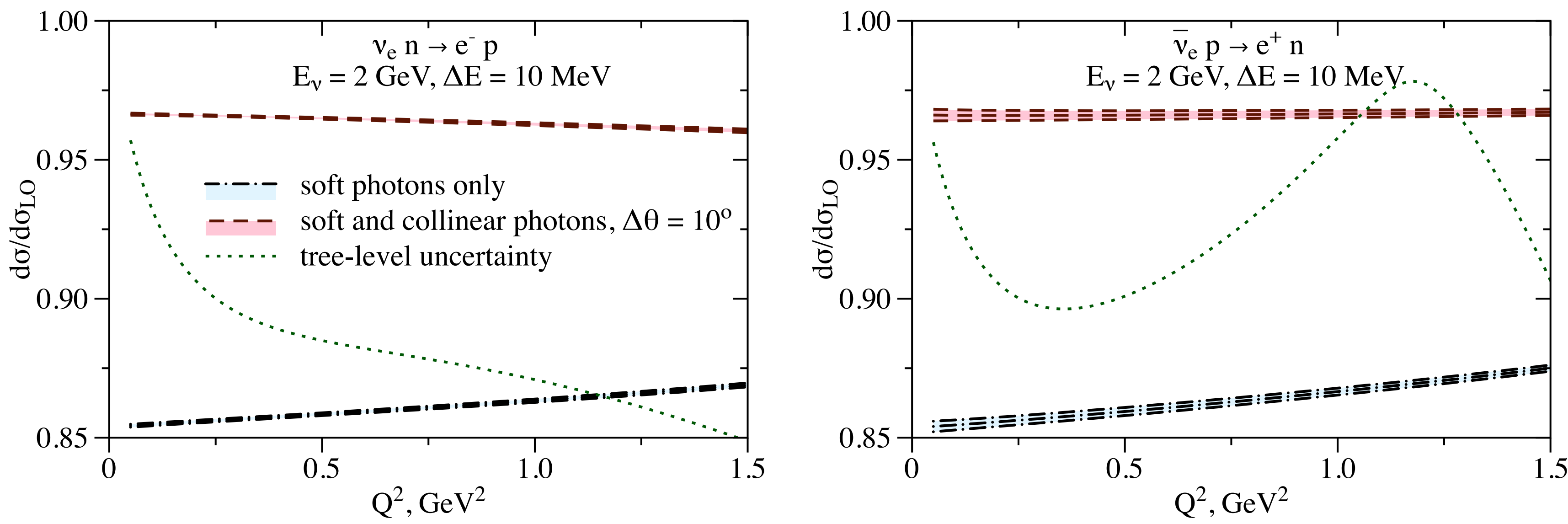

- ratio to LO: cancellation of uncertainty from hard function 


\section{Cross section. Muon flavor}

- exclusive observables
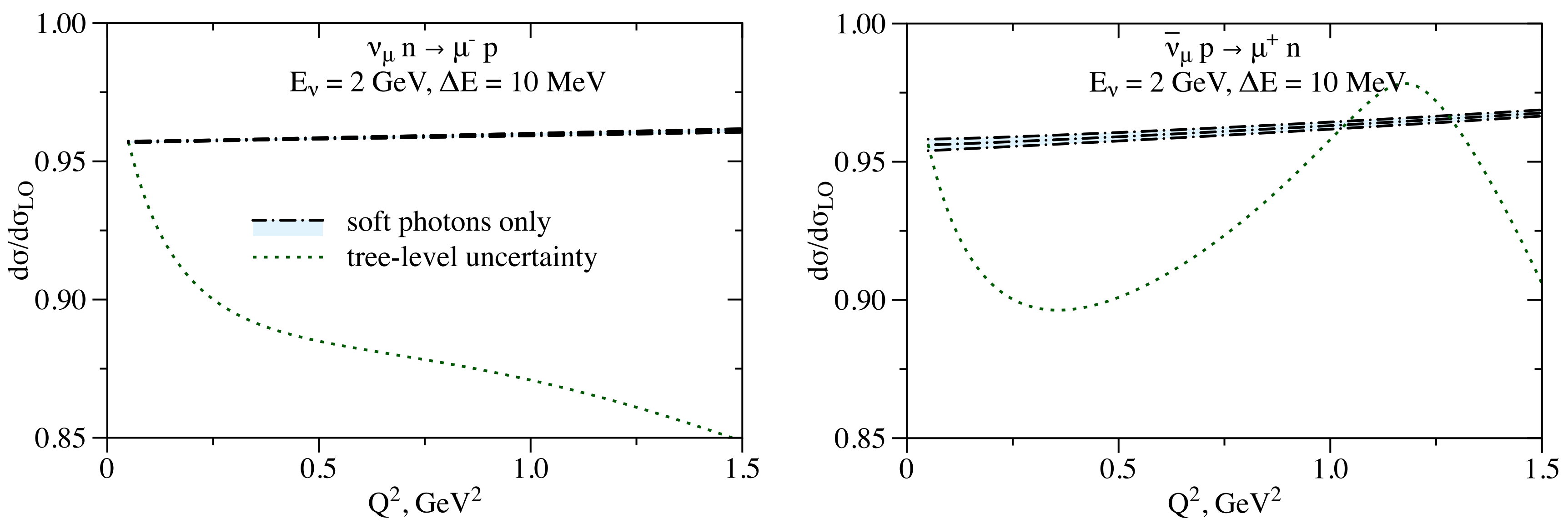

- ratio to LO: cancellation of uncertainty from hard function 


\section{Electron/muon ratio}

- exclusive observables
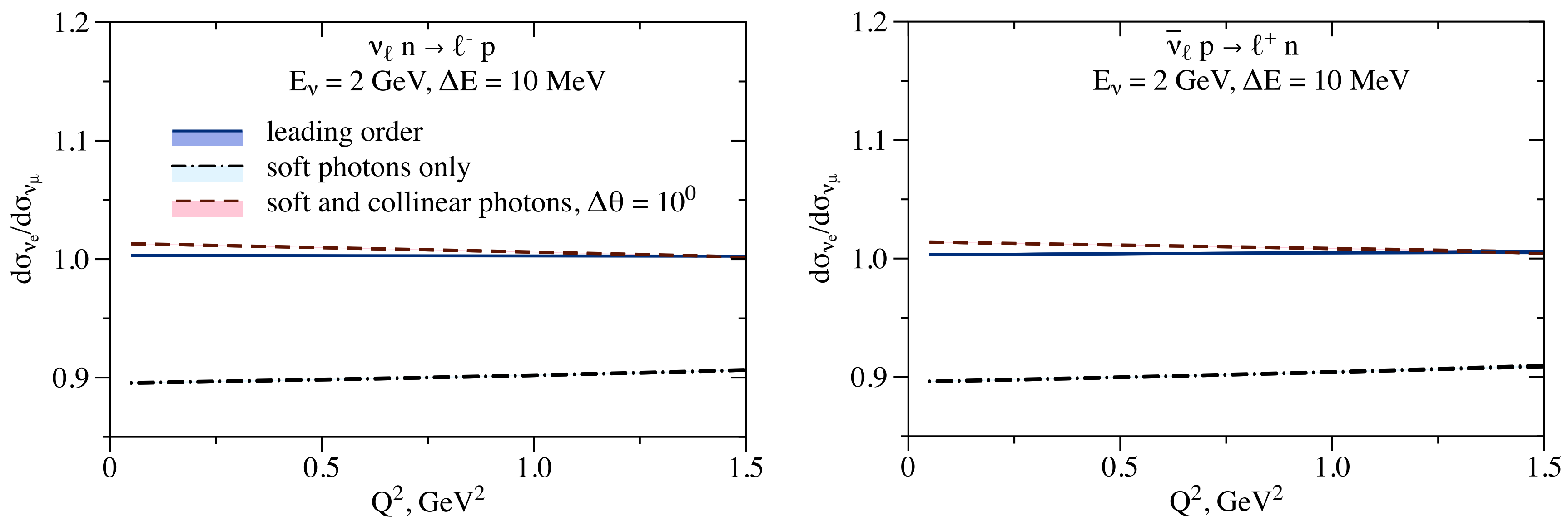

- small uncertainty: hard function does not depend on mass 


\section{Conclusions}

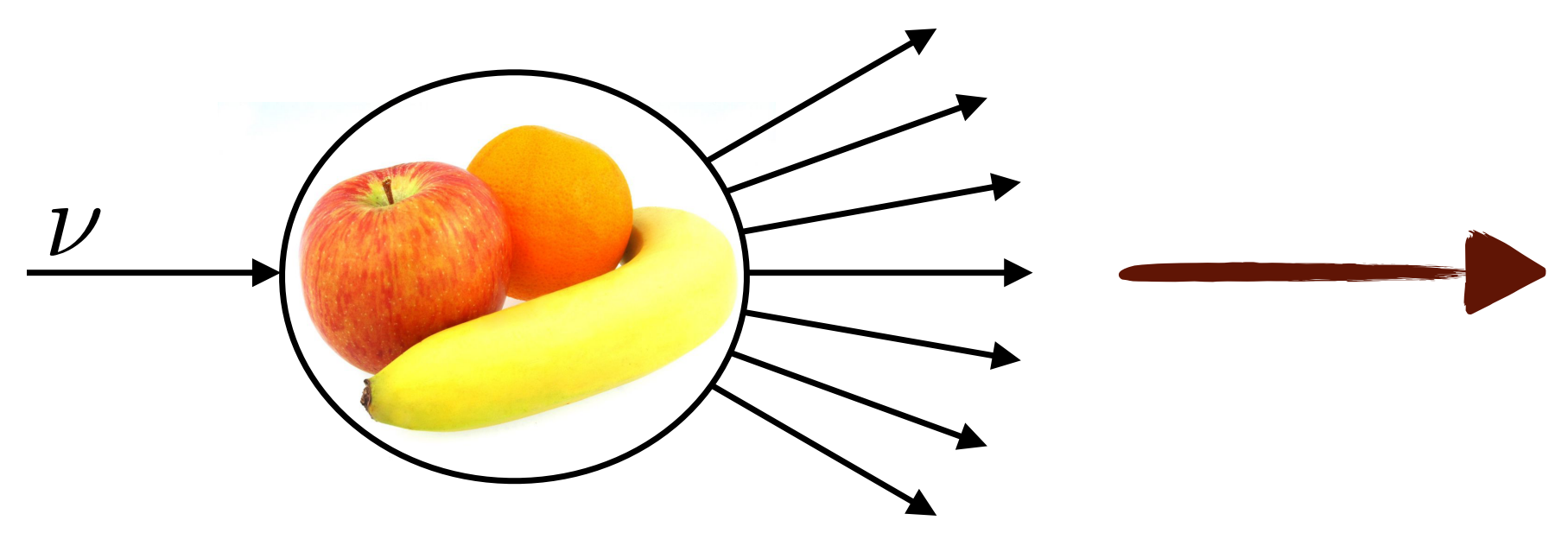

radiative corrections in EFT framework

- radiative corrections to neutrino-nucleon cross sections in factorization framework

- model for the hard function

- $\sigma_{\nu_{e}}$ can be predicted from $\sigma_{\nu_{\mu}}$ measurements with neutrino beam 


\section{Thanks for your attention !!!}

